www.nature.com/clinicalpractice/onc

human endothelial cells derived from lungs, aortic smooth muscle cells and human umbilical vein endothelial cells. The activity of this drug combination was also assessed using an in vivo mouse tumor model.

Paclitaxel and SU6668 were found to act synergistically to inhibit proliferation of vascular endothelial and smooth muscle cells in vitro, and strongly inhibited angiogenesis in MATRIGEL $®$ plugs (Discovery Labware Inc., Bedford, MA) implanted subcutaneously in mice. The combination also had a much greater apoptotic effect on endothelial cells than either agent alone. SU6668 alone had only marginal effect on the growth of a mouse xenograft of 1A9-PTX22, a paclitaxel-resistant human ovarian carcinoma. The combination of SU6668 with paclitaxel, however, delayed tumor growth and affected angiogenesis in the graft.

The authors state that rationally designed combination therapies have the potential to greatly improve cancer treatment by selectively targeting the various cell types and interactions involved in tumor growth.

Jim Casey

Original article Naumova E et al. (2006) The vascular targeting property of paclitaxel is enhanced by SU6668, a receptor tyrosine kinase inhibitor, causing apoptosis of endothelial cells and inhibition of angiogenesis. Clin Cancer Res 12: 1839-1849

\section{Expression of stromal MMPs affects clinical outcome in ovarian carcinoma}

Matrix metalloproteinases (MMPs) have a key role in the invasion and metastasis of a variety of tumors. Researchers have investigated whether epithelial (tumor-cell-derived) and stromal (host-derived) MMPs can be used to predict clinical outcome in patients with epithelial ovarian carcinoma (EOC).

Samples from 90 patients with EOCs were immunohistochemically stained for MMP-2, MMP-9 and MT1-MMP. High protein expression levels of any of the three stromal MMPs or epithelial MT1-MMP was associated with high tumor stage, high tumor grade, presence of ascites, and positive lymph node status. High levels of epithelial MMP-2 correlated only with positive lymph node status; high epithelial MMP-9 expression was not associated with any of the prognostic variables. In total, 55 deaths occurred, 52 of which were attributable to EOC. High epithelial or stromal expression of any of the three MMPs was associated with shorter median disease-specific survival. Multivariate analyses showed that high tumor stage, high expression of stromal MMP-9 and high expression of epithelial or stromal MT1-MMP were independently associated with poorer disease-specific survival.

The study indicates that high expression of stromal MMP-9 or MT1-MMP is associated with poor prognosis in patients with EOC. The authors state that further study is needed but that epithelial and stromal MMPs might be important targets for future ovarian cancer therapies.

Rebecca Ireland

Original article Kamat AA et al. (2006) The clinical relevance of stromal matrix metalloproteinase expression in ovarian cancer. Clin Cancer Res 12: 1707-1714

\section{Concomitant adjuvant chemoradiotherapy effective in node-positive breast cancer}

According to a French study, concomitant adjuvant chemoradiotherapy results in improved locoregional control and a shorter treatment period compared with sequential adjuvant chemoradiotherapy, when used in the treatment of patients with operable node-positive breast cancer who have previously undergone surgery.

Patients in this phase III, multicenter trial were randomized to receive four cycles of adjuvant 5-fluorouracil, mitoxantrone and cyclophosphamide with concomitant radiotherapy $(n=324)$, or four cycles of 5-fluorouracil, epirubicin and cyclophosphamide followed by radiotherapy 21 days later $(n=314)$. Patients were followed up for a median of approximately 5 years.

The risk of locoregional recurrence was decreased in the concomitant arm compared with the sequential arm, possibly because of the shorter interval between radiotherapy and initial surgery; the difference was significant in patients who had undergone lumpectomy (2.8-fold reduction, $P=0.027$ ). Additionally, the median treatment duration was approximately $50 \%$ less in the concomitant arm. Neither disease-free nor overall survival, however, were significantly different between the two arms. The

\section{GLOSSARY}

MATRIGEL $\odot$

The trade name for a gelatinous protein mixture secreted by mouse tumor cells, which because of its resemblance to the extracellular matrix is used as a tissue-culture substrate 